\title{
Altered sympathovagal balance and pain hypersensitivity in TNBS-induced colitis
}

\author{
Katarzyna Ciesielczyk, Agata Furgała, Łukasz Dobrek, Kajetan Juszczak, Piotr Thor
}

Department of Pathophysiology, Jagiellonian University Medical College, Krakow, Poland

Submitted: 20 October 2014

Accepted: 19 February 2015

Arch Med Sci 2017; 13, 1: 246-255

DOI: 10.5114/aoms.2015.55147

Copyright $\odot 2016$ Termedia \& Banach

\section{Abstract}

Introduction: Pain hypersensitivity, abnormal motility and autonomic dysfunction contribute to functional symptoms of inflammatory bowel disease (IBD). Material and methods: The aim of this study was to assess: nociceptive thresholds for mechanical allodynia (MA) and thermal hyperalgesia (TH), intestinal motility (distal colonic transit and emptying), and cardiac autonomic neuropathy (indices of heart rate variability - HRV) in male Wistar rats with experimental trinitrobenzene sulfonic acid (TNBS) induced colitis. To identify a potential vagal contribution the bilateral subdiaphragmatic vagotomy (SDV) was performed.

Results: Experimental colitis resulted in a significant decrease in pain threshold (MA $23.60 \pm 2.12, p<0.001$, TH $8.51 \pm 1.49, p<0.001$ ), reduced expulsion time $(6.2 \pm 3.5, p<0,01)$ and increase in the sympathetic autonomic activity (LFnu $32.54 \pm 21.16, p<0.03$ ). The animals with diminished vagal integrity presented with reduced gastrointestinal motility $(39.8 \pm 25.1$, $p<0.01)$ and a decrease in the parasympathetic high-frequency domain of HRV (HFnu $55.37 \pm 22.80, p<0.002$ ). The vagotomized rats with colitis showed the strongest nociceptive response (MA $22.46 \pm 3.02, p<0.004$; TH $7.99 \pm 1.12$, $p<0.003)$ as well as significant changes in sympatho-vagal balance on HRV testing (LFnu $28.25 \pm 14.66, p<0.04$; HFnu $71.34 \pm 14.55, p<0.04$ ).

Conclusions: The relationship between the cardiovascular and gastrointestinal system is modulated by neural, hormonal and inflammatory factors. This leads to dysregulation of the brain-gut interactions in the course of IBD. Sensitization and visceral-somatic convergence trigger pain hypersensitivity and autonomic sympathovagal imbalance. While integral vagal innervation impacts analgesic mechanisms via modulation of the immune response, SDV raises sympathetic activity and induces excessive hyperalgesia.

Key words: inflammatory bowel disease, colitis, pain hypersensitivity, heart rate variability.

\section{Introduction}

Pain hypersensitivity is a biological marker of gastrointestinal disorders and one of the common complaints of patients with inflammatory bowel disease (IBD). Abnormal endogenous pain modulation and visceral and somatic hypersensitivity may be accompanied by allodynia and hyperalgesia, due to viscerosomatic convergence of afferent pathways at the spinal cord level and within higher centers of the central nervous system (CNS) [1].

Several mechanisms, such as inflammation, post-injury sensitization, exposure to psychological or environmental stress and genetic predis-

\author{
Corresponding author: \\ Katarzyna Ciesielczyk PhD \\ Department \\ of Pathophysiology \\ Jagiellonian University \\ Medical College \\ 18 Czysta St \\ 31-121 Krakow, Poland \\ Phone: +48 607904634 \\ E-mail: ckatarzyna@op.pl
}


position, are postulated to be involved in IBD etiology [2, 3]. The brain-gut axis is controlled by intrinsic enteric nervous system (ENS) and extrinsic innervation originating from sympathetic and parasympathetic divisions of the autonomic nervous system (ANS). The spectrum of neurological manifestations of Crohn's disease (CD) and ulcerative colitis (UC) is reported in $25 \%$ to $50 \%$ of patients [4, 5]. The neuropathies accompanying IBD may result from extra-intestinal inflammation, immune-mediated disorders, nutritional imbalances (malabsorption, weight loss, vitamin deficiencies), or be an effect of administered medications [6]. The manifestations of peripheral neuropathies include mainly muscle weakness, sensory loss, presence of positive sensory symptoms and pain. In addition to peripheral neuropathy, IBD patients may show signs of autonomic impairment. Excess inflammatory response results in structural alternations of the autonomic innervation of the gut, such as axonal necrosis and altered size and number of the ganglia, which is reflected by profound impairment of intestinal motility and secretion.

Visceral information transmitted via the ANS afferents is integrated within the CNS, which results in the autonomic, endocrine, immune, motor and behavioral responses [7]. Sympathetic innervation derives from neurons located in the thoracic and lumbar spinal cord segments, and parasympathetic nerves originate from neurons located in the dorsal motor nucleus (DMN) of the vagus nerve (VN) and sacral parasympathetic nucleus of the spinal cord.

The neurovisceral integration and presence of the neuronal network may be reflected by heart rate variability (HRV). However, interpretation of such autonomic changes may be challenging, as many IBD patients undergo colectomy or small bowel resection, which may have a major impact on their fluid homeostasis. The evidence from previous studies on the occurrence of autonomic abnormalities in IBD patients is inconclusive. The authors of one study observed impairment of parasympathetic cardiac modulation in patients with UC and increased sympathetic activity in individuals with CD [8]. However, another study showed that both UC and CD are associated with increased vagal modulation [9]. According to most clinical studies, patients with CD are characterized by more prominent sympathetic involvement and individuals with UC present with more pronounced cardiovagal dysfunction.

Studies in animal models demonstrated that autonomic imbalance contributes to the inflammatory drive of experimental colitis. For example, both sympathectomy and administration of a parasympathomimetic nicotinic receptor agonist that improves colitis were shown to alleviate the symptoms of experimentally induced colitis [10]. The mechanisms of colonic inflammation were studied in animal models of IBD, such as colitis induced by intrarectal administration of TNBS (2,4,6-trinitrobenzene sulfonic acid). This model fairly well replicates the inflammatory component of human disease due to presence of a delayed type hypersensitivity reaction involving a T-helper type-1 immune response against hapten-modified autologous protein/luminal antigens.

The aim of this study was to analyze the relationship between pain hypersensitivity and ANS dysfunction observed in the course of TNBS-induced colitis. Nociceptive responses were assessed with behavioral tests and autonomic dysfunction was determined on the basis of HRV analysis. In order to evaluate the effect of vagal integrity we compared the results obtained in non-vagotomized and vagotomized rats with experimental TNBS-induced colitis. Determination of HRV is a non-invasive ECG-based technique used for the assessment of autonomic imbalances underlying various GI tract disorders; however, the available data on HRV in animals with experimentally induced IBD are fairly limited.

\section{Material and methods}

\section{Animals and procedures}

Male Wistar rats (230-260 g) were housed under standard conditions, kept in polycarbonate cages in temperature-controlled rooms with a 12-h light/dark cycle and access to standard rat chow and water ad libitum. The animals underwent acclimatization to the experimental environment, and then were handled regularly for 5 days prior to the experiments. During this time, they were habituated to plexiglass boxes and restrainers that were used later in the experiments, to eliminate any potential stress effects.

Prior to each experiment which required anesthesia, the animals were given an intraperitoneal injection of pentobarbital (30-50 mg/kg body weight, b.w.). Before each test, the animals were food deprived for $12 \mathrm{~h}$ with free access to water. After completing the experiments, the rats were euthanized via an overdose of pentobarbital, and their colon was removed for macroscopic examination. The protocol of the study was in agreement with the guidelines for the proper use of animals in biomedical research, and was granted approval from the Local Ethics Committee.

Induction of colitis: anesthetized rats received an enema of trinitrobenzenesulfonic acid (TNBS) 50 mg/kg b.w., 0.5 ml, 30\% EtOH (Sigma-Aldrich). TNBS was instilled into the colonic lumen via a flexible catheter (ID $2 \mathrm{~mm}$ ) inserted per rectum up to $7 \mathrm{~cm}$ proximally to the anus. To minimize 
the loss of administered liquid, the rats' hindquarters were elevated until they regained consciousness.

Subdiaphragmatic vagotomy (SDV): after midline laparotomy was performed, the stomach and lower esophagus were retracted in order to expose the ventral and truncal branches of the subdiaphragmatic vagus nerves which were eventually cut. The procedure was performed 7 days before the induction of TNBS-colitis in order to enable the animals to recover. Seven days following the procedure, one group of rats received saline enema (SDV rats), while TNBS enema was given to animals from the other group (SDV + TNBS rats). The completeness and effectiveness of the vagotomy were verified during postmortem inspection, on the basis of marked gastric dilation.

Histological examination: colonic specimens from the segment located 6 to $7 \mathrm{~cm}$ proximal to the rectum were fixed in $4 \%$ paraformaldehyde/ PBS, embedded in paraffin, cut into $3-\mu m$ thick sections, and subjected to routine hematoxylin and eosin (H\&E) staining. The slides were examined by a pathologist, who was blinded with regards to the animal assignment to a given group. The degree of colon damage was scored according to the following criteria [11]: 0 - no signs of inflammation, 1 - low degree of leukocyte infiltration, 2 - moderate degree of leukocyte infiltration, 3 - high degree of leukocyte infiltration, high vascular density and thickening of bowel wall, 4-transmural infiltrations, loss of goblet cells, high vascular density and thickening of the bowel wall.

\section{Behavioral nociceptive tests}

Behavioral reflex responses (Plantar tests - Ugo Basile, Italy) were assessed 7 days after administration of TNBS, or saline. The tests were conducted by a person who was blinded with regards to the animal assignment to a given group.

Mechanical allodynia - von Frey test: Punctate mechanical hypersensitivity was assessed using the electronic von Frey device. The rats were placed on a wire mesh floor in a plastic enclosure. A calibrated force transducer (with a $0.5 \mathrm{~mm}^{2}$ tip) was applied with increasing pressure (up to $26 \mathrm{~g}$ ) onto the mid-plantar surface of the hind paw until an active limb withdrawal response was observed. The paw withdrawal threshold (PWT) was calculated as a mean value from three consecutive measurements.

Thermal hyperalgesia - Hargreaves test: The response to infrared noxious heat stimuli was examined. The animals were placed in a clear plexiglass box with a dry glass floor. A focused beam of radiant heat with a constant temperature of $46^{\circ} \mathrm{C}$ and $50-\mathrm{nm}$ wavelength was applied onto the plantar surface of the paw. The hind PWL to the thermal stimulus was calculated as the mean value from three consecutive measurements.

\section{Colonic propulsion test}

Distal colonic transit and emptying were assessed using a single 3-mm glass bead which was inserted per rectum, up to $2 \mathrm{~cm}$ inside the distal colon. The rats were placed in individual plastic cages and the time of bead expulsion was noted. A long expulsion time was considered a marker of delayed colonic propulsion.

\section{ECG recordings and HRV data analysis}

The ECG recordings were obtained with LabChart v7.3 (ADInstruments) in animals under anesthesia. A typical output is shown in Figure 1. The standard three-lead ECG was used. The animals were examined in a supine position, with stable body temperature maintained with a warming pad with temperature feedback control function (Homeothermic Controller and Plate). The RR interval files were generated from 20-min ECG segments after their careful visual inspection for any artifacts that could affect the outcome. The HRV analysis of standard time- and frequency-domain indices was conducted in line with European Society of Cardiology guidelines.

Time-domain analysis: indices included values of mean R-R intervals (mRR) and mean heart rate (HR).

Frequency-domain analysis: power spectral density was determined by applying the fast Fourier transform (FFT), calculated by using 1024 points and half overlap with a Welch window. Cutoff frequencies divided the power spectrum into two main parts, LF - low frequency $(0.04-1.0 \mathrm{~Hz})$ and $\mathrm{HF}$ - high frequency $(1.0-3.0 \mathrm{~Hz})$, as previously described $[12,13]$.

The LF and HF components are expressed in normalized units (nu), which represent the relative value of each power component in proportion to the total power minus the very low-frequency component (VLF 0.0-0.4 Hz). Expression in normalized units can minimize the effects of the changes in total power on the values of the LF and HF components, and is recommended especially when changes in HF and LF components are compared in the same subject.

\section{Experimental protocol and statistics}

Four groups of rats (each $n=10$ ) were subjected to experiments: 1 - control - saline treated, 2 - TNBS-treated rats, 3 - SDV - rats subjected to subdiaphragmatic vagotomy and given saline enema 7 days after the surgery, 4 - SDV + TNBS - rats subjected to subdiaphragmatic vagotomy and given TNBS enema 7 days after the surgery. All the tests and measurements were performed 


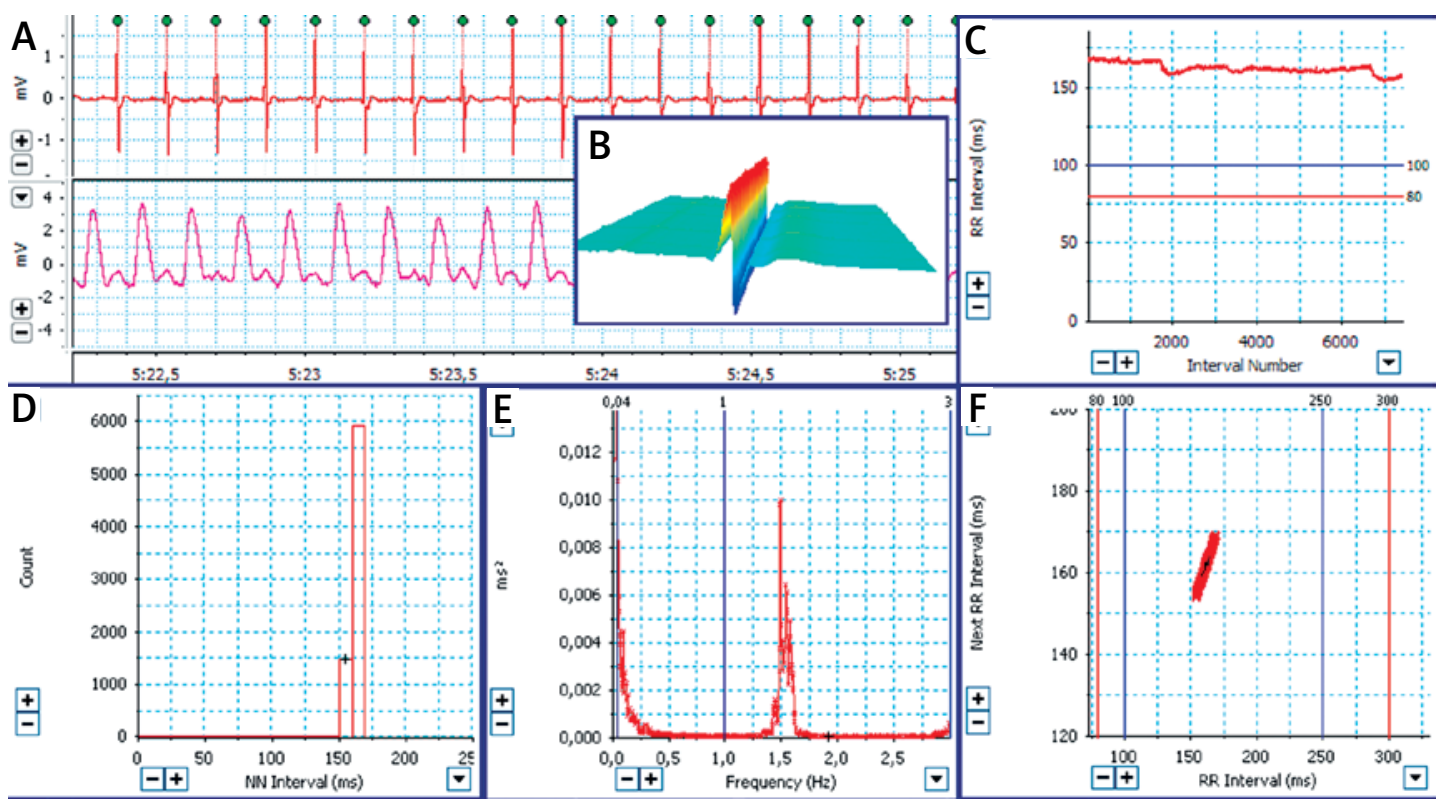

Figure 1. Output from HRV analysis program Chart (ADInstruments) under basal conditions. A - original ECG recording; B - waterfall plot of ECG; C - tachogram - RR point series; D - histogram displaying normalized RR intervals; E - spectrum - time series after resampling, power spectral density (PSD), F - Poincare Plot - each RR interval plotted against the preceding RR interval. Parameters in the time domain were determined on the original tachogram. Parameters in the frequency domain: the power spectral density was calculated by applying the fast Fourier transform (FFT) that was sampled and $1000 \mathrm{~Hz}$ and calculated by using 1024 points and half overlap with a Welch window. Cutoff frequencies divided the power spectrum into two main parts, $\mathrm{LF}-$ low frequency $(0.04-1.0 \mathrm{~Hz})$ and $\mathrm{HF}$ - high frequency $(1.0-3.0 \mathrm{~Hz})$

at baseline and 7 days following the administration of TNBS or saline.

\section{Statistical analysis}

Statistical analysis of the results was conducted with Statistica v. 10 software. A paired $t$-test was used to compare the results of the control and TNBS rats, as well as for SDV rats vs. SDV + TNBS rats comparison. Additionally, the results of the controls were compared to those of SDV rats and SDV + TNBS rats using the paired $t$-test. The results are presented as mean \pm standard deviation (SD), and were considered significant at a $p$-value $\leq 0.05$. ${ }^{\star} p$ denotes significant differences compared to the controls and ${ }^{\#} p$ significant differences compared to SDV rats.

\section{Results}

\section{Behavioral nociceptive tests}

The analysis of hind paw hypersensitivity resulting from stimulation during the von Frey test revealed that the rats from all three investigated groups (TNBS, SDV, SDV + TNBS) showed markedly greater responsiveness to mechanical stimulation than the controls. The TNBS-treated rats, especially those subjected to vagotomy SDV + TNBS, presented with particularly low PWT. The PWT of the SDV + TNBS rats was significantly lower than that of SDV (Figure $2 \mathrm{~A}$, Table I).
Both the TNBS rats and the SDV + TNBS rats showed greater hypersensitivity to thermal stimulation of the hind paw during the Hargreaves test. The SDV + TNBS rats presented with significantly shorter PWL than did the SDV rats. The SDV group did not show thermal hypersensitivity (Figure 2 B, Table I).

\section{Colonic propulsion test}

Colonic propulsion was estimated on the basis of time required for expulsion of the glass bead. The expulsion time of the SDV rats turned out to be significantly longer than that of the controls, which pointed to inhibition of colonic propulsion. The comparative analysis of expulsion times (TNBS rats vs. controls and TNBS + SDV rats vs. SDV rats) showed that the TNBS-induced inflammation resulted in a marked decrease in colonic propulsion (Figure $2 \mathrm{C}$, Table I).

\section{Heart rate variability}

The values of baseline HRV parameters determined in each group of rats are presented in Table II and Figure 3. The SDV + TNBS rats presented with a significantly higher heart rate (HR) than the vagotomized animals (SDV rats). Mean RR and total power (TP) followed a similar pattern: the values of these parameters were higher in the SDV rats than in the controls, and markedly lower in the SDV + TNBS rats than in the SDV rats. 
A

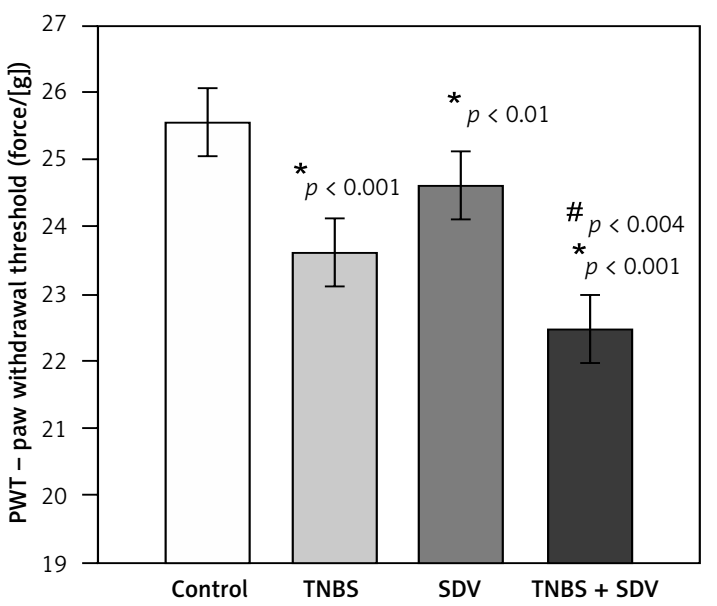

C

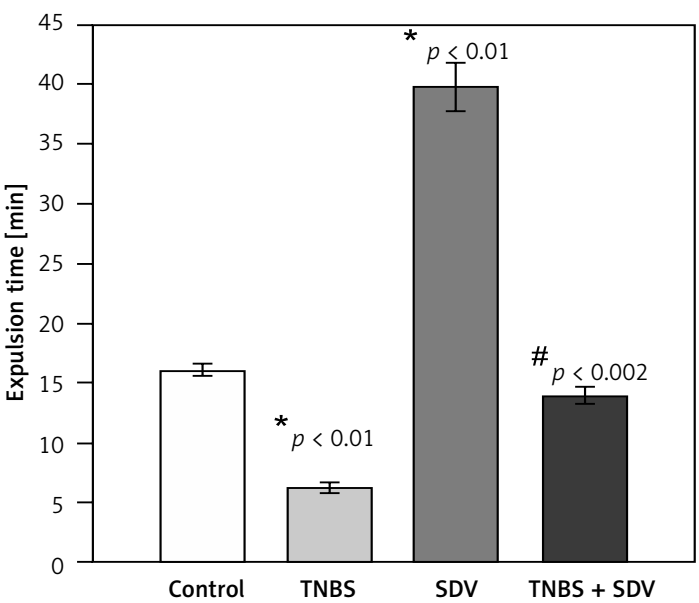

B

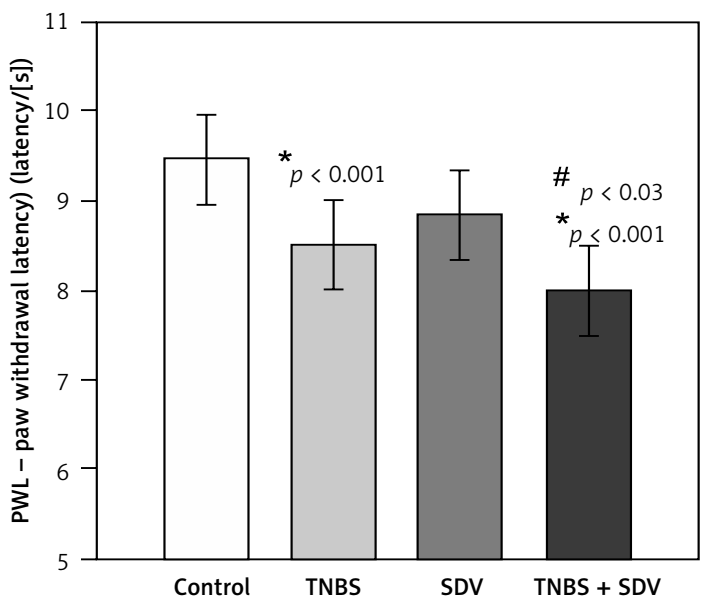

Figure 2. Results of behavioral nociceptive tests and colonic propulsion test in groups: control, TNBS-treated rats, SDV - rats after subdiaphragmatic vagotomy, SDV + TNBS - vagotomized TNBStreated rats: $\mathrm{A}$ - mechanical allodynia threshold $[\mathrm{g}]$ that elicited hind paw withdrawal - PWT, B - thermal hyperalgesia threshold [s] that elicited hind paw latency - PWL, C - colonic propulsion test expulsion time [min]

${ }^{*} p$ vs. control, $" p$ vs. SDV.

Table I. Results of behavioral nociceptive tests and colonic propulsion test in groups: control, TNBS-treated rats, SDV - rats after subdiaphragmatic vagotomy, SDV + TNBS - vagotomized TNBS-treated rats; all values are means \pm standard deviation. Mechanical allodynia threshold [g] that elicited hind paw withdrawal - PWT. Thermal hyperalgesia threshold [s] that elicited hind paw latency - PWL (see Figures $2 \mathrm{~A}, \mathrm{~B}$ ). Colonic propulsion test - the time required for expulsion of the glass bead [min]

\begin{tabular}{|lcccc|}
\hline Variable & Control & TNBS & SDV & SDV + TNBS \\
\hline $\begin{array}{l}\text { Behavioral nociceptive tests: } \\
\text { Mechanical allodynia - PWT [g] }\end{array}$ & $25.55 \pm 0.74$ & $23.60 \pm 2.12^{*}$ & $24.62 \pm 2.27^{*}$ & $22.46 \pm 3.02^{\star \#}$ \\
\hline Thermal hyperalgesia - PWL [s] & $9.44 \pm 1.39$ & $8.51 \pm 1.49^{*}$ & $8.85 \pm 1.51$ & $7.99 \pm 1.12^{\star \#}$ \\
\hline Colonic propulsion test - time [min] & $16.0 \pm 8.1$ & $6.2 \pm 3.5^{*}$ & $39.8 \pm 25.1^{*}$ & $13.9 \pm 7.9^{\#}$ \\
\hline
\end{tabular}

${ }^{*} p$ vs. control, " $p$ vs. SDV.

The analysis of the parameters of sympathetic and parasympathetic function revealed that vagotomy resulted in a significant decrease in vagal tone (HFnu), and caused an increase in sympathetic tone (LFnu) and cardiac sympatho-vagal ratio (LF/HF).

Sympathetic activation plays an important role in the pathogenesis of inflammation; this was confirmed by the fact that the TNBS rats presented with significantly higher values of LFnu and LF/ $\mathrm{HF}$ ratio, and lower values of HFnu than did con- trols. The SDV + TNBS rats showed a markedly higher level of the parasympathetic tone (HFnu) and lower values of the sympathetic tone (LFnu) and LF/HF ratio when compared to the SDV rats.

\section{Assessment of inflammatory severity, vagotomy and body weight changes}

Exposure to TNBS resulted in moderately severe chronic colitis, mainly involving the cecum and proximal colon. The rats with TNBS-induced colitis showed 
Table II. Autonomic nervous system test - heart rate variability (HRV) parameters in groups: control, TNBS-treated rats, SDV - rats after subdiaphragmatic vagotomy, SDV + TNBS - vagotomized TNBS-treated rats; all values are means \pm standard deviation

\begin{tabular}{|c|c|c|c|c|}
\hline Variable & Control & TNBS & SDV & SDV + TNBS \\
\hline \multicolumn{5}{|c|}{ Autonomic nervous system test - heart rate variability (HRV): } \\
\hline $\mathrm{HR}[\mathrm{bpm}]$ & $350.74 \pm 26.22$ & $367.02 \pm 35.26$ & $323.34 \pm 33.67$ & $356.93 \pm 17.92^{\ddagger}$ \\
\hline $\mathrm{mRR}[\mathrm{ms}]$ & $171.92 \pm 12.60$ & $166.39 \pm 15.92$ & $197.11 \pm 32.60^{*}$ & $168.48 \pm 8.37^{\#}$ \\
\hline $\mathrm{TP}\left[\mathrm{ms}^{*} \mathrm{~ms}\right]$ & $1.73 \pm 0.80$ & $1.721 \pm 0.77$ & $3.62 \pm 2.66^{*}$ & $1.74 \pm 0.57^{\#}$ \\
\hline LFnu & $17.04 \pm 8.74$ & $32.54 \pm 21.16^{\star}$ & $44.24 \pm 23.37^{\star}$ & $28.25 \pm 14.66^{\text {*\# }}$ \\
\hline HFnu & $82.71 \pm 8.74$ & $65.83 \pm 20.17^{\star}$ & $55.37 \pm 22.80^{*}$ & $71.34 \pm 14.55^{*} \#$ \\
\hline $\mathrm{LF} / \mathrm{HF}$ & $0.22 \pm 0.14$ & $0.68 \pm 0.67^{*}$ & $1.02 \pm 0.80^{*}$ & $0.46 \pm 0.38^{\#}$ \\
\hline
\end{tabular}

$H R$ - mean hear rate, $m R R$ - mean RR intervals, TP - total power, LFnu - low frequencies normalized, HFnu - high frequencies normalized, $L F / H F$ ratio, " $p$ vs. control, " $p$ vs. SDV (see Figure 3).

postural and behavioral changes, including repeated licking of the lower abdomen. Overall condition of the animals was assessed on the basis of relative change in their body weight (in \%) between treatment day one and seven. The weight gain of the TNBS rats was significantly lower than that of the controls (weight gain $2 \%$ vs. $21 \%$, respectively). Contrary to the SDV rats, no changes in body weight were documented in SDV + TNBS rats ( $11 \%$ vs. $0 \%$ respectively).

On macroscopic examination, the cecum appeared edematous, with mild erythema, sporadic ulcers and erosions. Microscopic examination showed that the changes found in the colonic wall of TNBS-treated rats were more advanced than those observed in the controls (mean score 3.1 vs. 0.5 , respectively). Moreover, the degree of microscopic changes present in the SDV + TNBS rats was higher than in the case of the vagotomized (SDV) rats (mean score 3.6 vs. 1 respectively) (Figure 4).

The SDV rats demonstrated impaired gastrointestinal motility and showed marked gastric dilation on postmortem examination. The result of the propulsion test pointed to prolonged colonic transit as a potential cause of chronic constipation observed in this group.

\section{Discussion}

The present study examined the relationship between pain hypersensitivity and autonomic nervous system regulation in an experimental model of inflammatory bowel disease. To understand the impact of hypersensitivity and ANS modulation on the course of the disease, we analyzed the following groups of rats: TNBS-treated rats, animals subjected to SDV, subjected to vagotomy followed by TNBS treatment, and controls.

\section{Visceral hypersensitivity}

During behavioral nociceptive tests the TNBS rats showed hypersensitivity to mechanical al- lodynia (von Frey test) and thermal hyperalgesia (Hargreaves test), manifested by a decreased pain threshold. However, an even stronger nociceptive response was observed in the case of the SDV + TNBS rats. These findings are consistent with the results of previous studies, in which stimulation of the abdominal wall or hind paw of animals with colonic inflammation resulted in enhanced nociceptive responses [14]. This phenomenon can be explained as a consequence of complex structural changes taking place in the ENS of IBD subjects. The myenteric plexus, located between longitudinal and circular muscle layers of the intestinal wall, is primarily involved in the regulation of motility, while the submucosal plexus in the submucosa is mainly responsible for control of secretory, absorptive, and vascular functions [15]. The ENS communicates with the CNS via sympathetic and parasympathetic efferent neurons, as well as via afferent neurons that run within parasympathetic and spinal nerve bundles. Inflammatory bowel disease was shown to be associated with plasticity of the ENS, as gastrointestinal specimens from patients with this condition display alterations of the enteric ganglia, such as neuronal hypertrophy and glial hyperplasia [16].

We analyzed visceral hypersensitivity on the basis of expulsion time of a glass bead inserted into the distal colon. The rats with TNBS-induced colitis presented with enhanced colonic motility, as shown by significantly shorter expulsion time of the bead. Colonic motility depends on the activity of the myenteric neurons, interstitial cells of Cajal (ICC), glial cells and smooth muscle cells [17]. The vagus nerve, capsaicin-sensitive fibers and sympathetic neural pathways are involved in the motor response of the colon. Noxious stimuli alter the sensitivity of the colonic afferent nerves, which is reflected by the perception of abdominal discomfort. Enterocytes release mediators, such as leukotriene B4, prostaglandins (PGE2) and tumor 
A

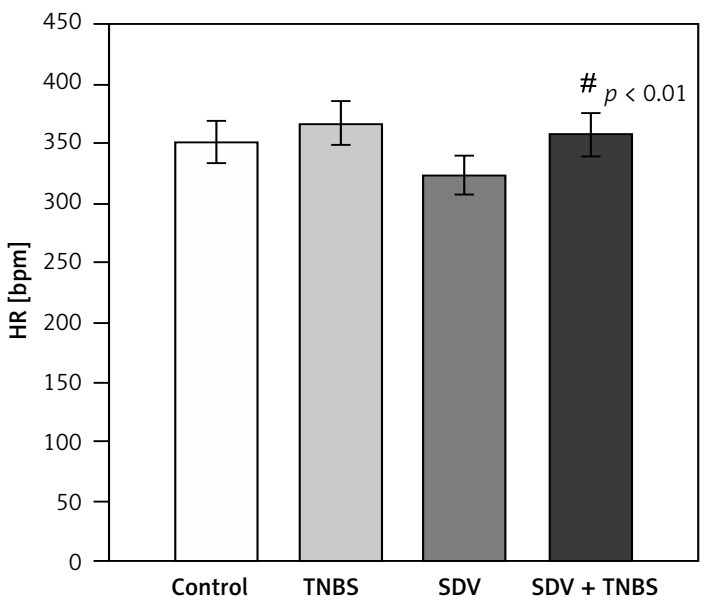

C

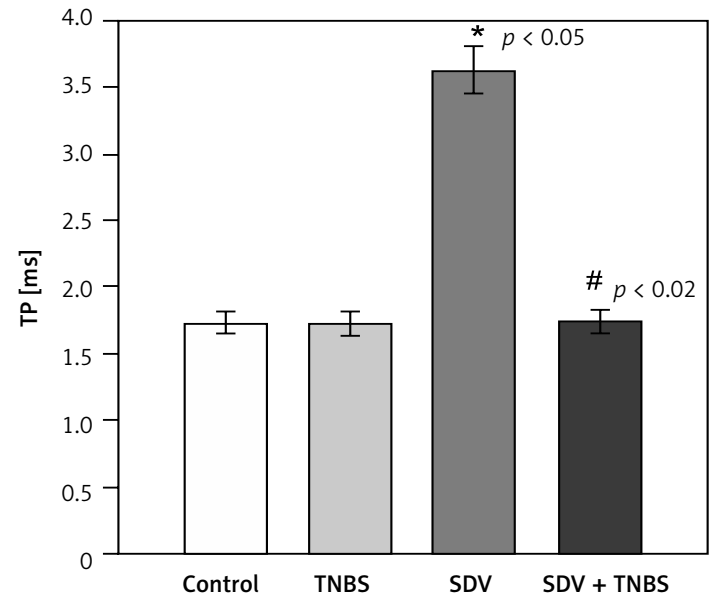

E

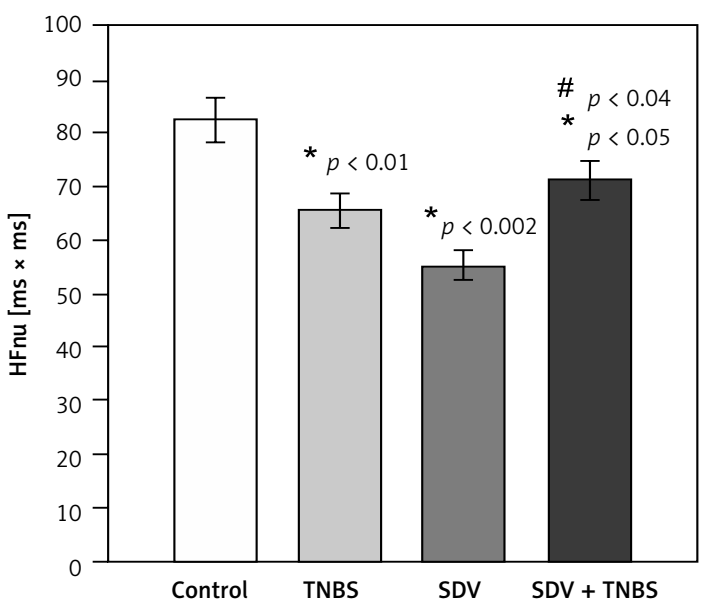

B

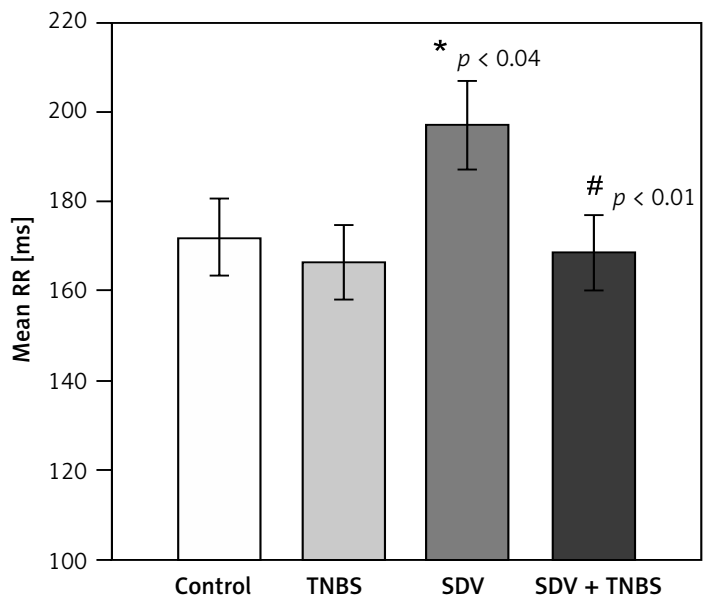

D

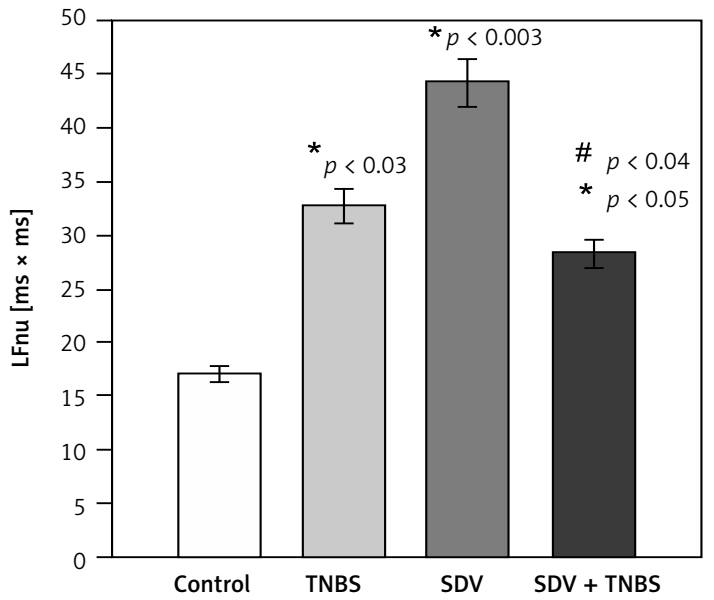

$\mathrm{F}$

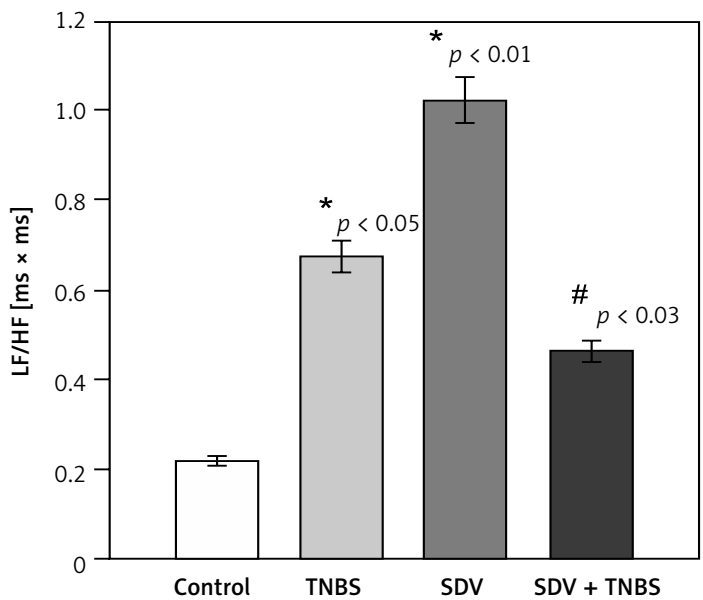

Figure 3. Autonomic nervous system test - results of heart rate variability (HRV) parameters in groups: control, TNBS-treated rats, SDV - rats after subdiaphragmatic vagotomy, SDV + TNBS - vagotomized TNBS-treated rats. A - heart rate (HR), B - mean RR intervals, C - total power (TP), D - low frequencies normalized (LFnu), E - high frequencies normalized (HFnu), F - LF/HF ratio

*p vs. control, " $p$ vs. SDV (see Table II).

necrosis factor $\alpha$ (TNF- $\alpha)$. Other mediators that are released include proinflammatory cytokines $(\mathrm{IL}-1 \beta$, IL-6) from monocytes and serotonin (5-HT) from enterochromaffin cells [18]. The excess of 5-HT enhances peristalsis and secretion of fluid and mucin; this may constitute a potential cause 
of the symptoms observed in the TNBS-treated rats, i.e. diarrhea and shorter expulsion time during the colonic propulsion test.

Peripheral inflammatory neuropathy causes rearrangement of synaptic contacts, wind-up, central sensitization, long-term potentiation and loss of inhibitory neurons of the spinal dorsal horn [19]. The central sensitization was attributed to the enhanced activity of the $C$ fibers; activation of these fibers is reflected by the release of glutamate, histamine and substance $P$, and the resultant increase in the excitability of their postsynaptic targets [20]. Furthermore, all these compounds modulate pain behavior, as observed during mechanical and thermal stimulation of the TNBS-treated rats.

In the present study, animals after surgical interruption of vagal innervation (SDV rats) showed significant inhibition of gastrointestinal peristalsis and prolonged expulsion time, contrary to the SDV + TNBS rats, whose expulsion time was markedly accelerated. These observations are consistent with the result of some previous studies showing that cholinergic neuronal remodeling, taking place during disruption of the subdiaphragmatic vagal nerves, results in decrease in colonic motility and its consequences, such as constipation and abnormal visceral perception [21, 22].

We found that induction of colitis in vagotomized rats resulted in a stronger increase in the threshold of the nociceptive response to mechanical allodynia and thermal hyperalgesia than in rats with induced colitis without prior vagotomy. These findings suggests that vagal tone modulates the inflammatory response and may protect against development of hypersensitivity associated with IBD. Previous studies showed that vagal innervation may attenuate inflammation, thus protecting against TNBS-induced colitis; in turn, vagotomy was shown to aggravate colitis [23]. According to some authors, vagotomy increases systemic production of TNF- $\alpha$, and selective inhibition of vagal afferent fibers by perivagal application of capsaicin results in an increase in the severity of hapten-induced colitis [24]. Also, stimulation of the vagus nerve was demonstrated to selectively downregulate the synthesis of pro-inflammatory cytokines [25].

The cholinergic anti-inflammatory pathway is involved in the efferent activity of the vagus nerve, leading to release of acetylcholine, which interacts with the $\alpha 7$-nicotinic receptor on macrophages. Activation of the nicotinic receptor leads to inhibition of the nuclear factor (NF)- $\kappa B$ pathway and a resultant decrease in the synthesis of pro-inflammatory cytokines (IL- 6 and TNF- $\alpha$ ) but not anti-inflammatory cytokines such as IL-10 [26]. Vagal activity modulates sympathetic tone

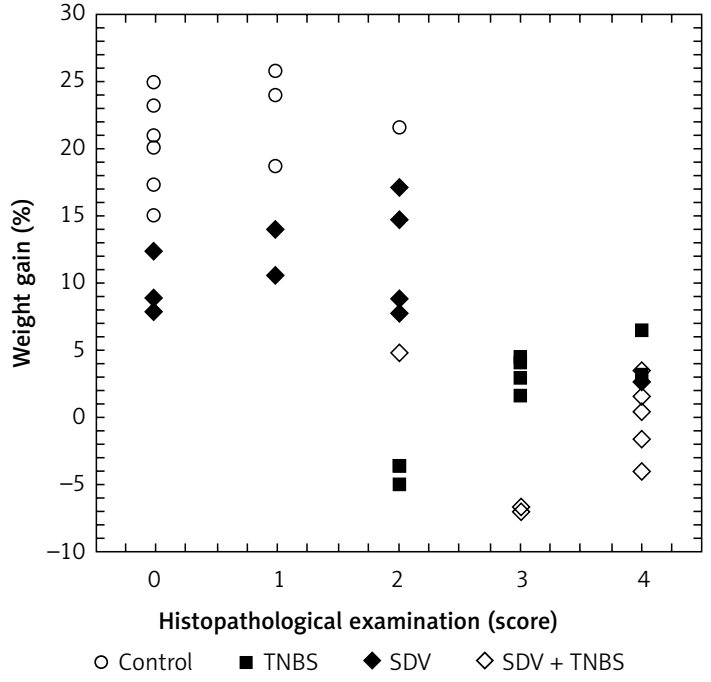

Figure 4. The relation between the percentage weight gain (from day 1 to 7 ) and the result of histological examination (colonic wall damage score 1 to 4, as described in the methods section) in groups: control, TNBS-treated rats, SDV - rats after subdiaphragmatic vagotomy, SDV + TNBS - vagotomized TNBS-treated rats

and reduces oxidative stress [27]. The neuroimmune feedback mechanism triggers a hormonal response, which leads to activation of the hypothalamic-pituitary-adrenal (HPA) axis and the sympatho-adrenomedullary system, resultant central release of corticotrophin-releasing factor (CRF) and peripheral glucocorticoid secretion by the adrenal cortex [28]. Cortisone affects a number of functions, including the immune response, metabolic processes and intestinal motility in IBD patients.

\section{Autonomic nervous system}

Fast Fourier transformation (FFT) was used to split HRV into high- and low-frequency (HF and LF) powers during frequency domain spectral analysis of ECG. The LF oscillations reflect both vagal and sympathetic input to the heart, but we used LFnu expressed in normalized units, which is a marker of sympathetic modulation [29]. The LF/HF ratio reflects sympathetic-parasympathetic nervous balance or sympathetic modulations. The HFnu components are induced by fluctuations in cardiac-vagal input and correspond to respiratory sinus arrhythmia controlled by medullary vagal centers, carotid-aortic baroreceptors and atrial stretch receptors [30]. While the sympathetic system enhances heart automaticity via the rostral ventrolateral medulla, the parasympathetic system inhibits it via the ambiguus and vagal dorsal motor nuclei, and reflex feedback constantly adjusts the impact of each system [31]. The ANS of IBD patients is altered both structurally and functionally; the struc- 
tural changes observed in the autonomic nerves of the gut include neuronal hyperplasia, ganglionic and axonal degeneration and necrosis. Recent studies showed that experimentally induced colitis stimulates expression of Fos in a specific subset of postganglionic sympathetic neurons of the inferior mesenteric ganglion that project to the colon. The evidence of autonomic imbalance is supported by the fact that chemical sympathectomy was shown to result in less damage, partial resolution of microscopic lesions and attenuation of symptoms. Furthermore, administration of a parasympathomimetic was shown to improve the course of colitis in an animal model [32].

Our findings provide an insight into the cardiovascular autonomic changes. We demonstrated that TNBS-induced inflammation is associated with a predominance of sympathetic tone, as shown by a decrease in mean R-R intervals, increase in LFnu and LF/HF ratio, and lower parasympathetic HFnu activity than in saline-treated controls.

Potential mechanisms underlying the sympathetic overactivity observed in TNBS-treated rats may be associated with local release of inflammatory mediators and resultant chronic stimulation of enteric visceral afferents, leading to abnormal afferent transmission to the spinal cord and brain stem. The overall increase in the sympathetic activity may also result from a decrease in plasma volume, as the active phase of UC is associated with diarrhea. Indeed, unloading of cardiopulmonary and arterial baroreceptor afferents observed in the course of plasma hypovolemia is reflected by a diminished central inhibitory modulation of efferent sympathetic activity, and the resultant increase in its discharge activity. This leads to vasoconstriction and increase in $\mathrm{HR}$, both aimed the maintenance of normal blood pressure [33].

The sympatho-vagal balance and the activity of the HPA axis are controlled by the hippocampus, prefrontal cortex and amygdala. The amygdala receives large inputs from the vagal nerve, which triggers its negative feedback on the HPA axis. The prefrontal cortex regulates peripheral immune cells via the autonomic and neuroendocrine pathways and controls the parasympathetic cholinergic anti-inflammatory outflow [34]. Furthermore, these regions are involved in the regulation of pain, emotional and cognitive behaviors.

Alterations of the sympathetic tone were found to facilitate and sustain the local inflammatory response in UC; moreover, both pain and psychological stress are known to reactivate quiescent UC [35]. It is postulated that pain may be modulated via a descending pathway, in line with the gate control theory. Descending projections from the anterior cingulate cortex (ACC) and amyg- dala to the pontomedullary networks, including the periaqueductal gray, rostral ventral medulla, and raphe nuclei, activate inhibitory opioidergic, serotoninergic, and noradrenergic pathways to the dorsal horn of the spinal cord. This acts like a "gate", increasing or decreasing projection of afferent impulses arising from peripheral nociceptive sites to the CNS [36].

We found that the blockade of vagal signaling due to surgical vagotomy resulted in an increase in mean RR and sympathetic activity (LFnu, LF/ HF) and, importantly, was reflected by a marked decrease in the parasympathetic high frequency power. This confirms that HRV is an accurate measure of non-cardiac activity. Vagotomized TNBS-treated rats showed higher values of HR and HFnu and lower LFnu and LF/HF levels than did the SDV animals. Moreover, vagotomy impaired the function of the mucosal barrier, which resulted in greater exposure of intestinal mucosa to TNBS and other factors present in the colonic lumen, e.g. bacterial antigens. A previous study showed that intestinal permeability is modulated by cholinergic nerves, and vagotomy was demonstrated to increase the permeability of rat intestine [37].

In conclusion, we demonstrated that TNBS-induced colitis is associated with profound changes in the autonomic sympathovagal balance, accelerated colonic propulsion and enhanced pain hypersensitivity. Subdiaphragmatic vagotomy resulted in a decrease of vagal tone as reflected by changes in selected HRV indices, longer expulsion time and constipation. Vagotomized rats with experimentally induced colitis presented with a more severe course of the disease and demonstrated the lowest pain threshold during nociceptive testing. Our future research will center on the activation of the cholinergic anti-inflammatory pathway due to electrical stimulation of the vagus nerve. Furthermore, we will search for novel methods of normalizing the IBD-specific autonomic abnormalities, which may serve as a potential basis for new treatment strategies of this condition.

\section{Conflict of interest}

The authors declare no conflict of interest.

\footnotetext{
References

1. Ciesielczyk K, Thor PJ. Neural control disturbances of the gastrointestinal tract and visceral pain in inflammatory bowel diseases. Postepy Hig Med Dosw 2013; 67: 304-14.

2. Kaser A, Zeissig S, Blumberg RS. Genes and environment: how will our concepts on the pathophysiology of IBD develop in the future? Dig Dis 2010; 28: 395-405.

3. Xu L, Dai WQ, Wang F, et al. Association of STAT4 gene rs7574865G > T polymorphism with ulcerative colitis risk: evidence from 1532 cases and 3786 controls. Arch Med Sci 2014; 10: 419-24.
} 
4. Zois CD, Katsanos KH, Kosmidou M, Tsianos EV. Neurologic manifestations in inflammatory bowel diseases: current knowledge and novel insights. J Crohns Colitis 2010; 4: 115-24.

5. Gondim FA, Brannagan TH, Sander HW, Chin RL, Latov N. Peripheral neuropathy in patients with inflammatory bowel disease. Brain 2005; 128: 867-79.

6. Kawalec P, Mikrut A, Wiśniewska N, Pilc A. Tumor necrosis factor-alpha antibodies (infliximab, adalimumab and certolizumab) in Crohn's disease: systematic review and meta-analysis. Arch Med Sci 2013; 9: 765-79.

7. Bonaz BL, Bernstein CN. Brain-gut interactions in inflammatory bowel disease. Gastroenterology 2013; 144: 36-49.

8. Lindgren S, Stewenius J, Sjolund K, Lilja B, Sundkvist G. Autonomic vagal nerve dysfunction in patients with ulcerative colitis. Scand J Gastroenterol 1993; 28: 638-42.

9. Mouzas IA, Pallis AG, Kochiadakis GE, et al. Autonomic imbalance during the day in patients with inflammatory bowel disease in remission. Evidence from spectral analysis of heart rate variability over 24 hours. Dig Liver Dis 2002; 34: 775-80.

10. Eliakim R, Karmeli F, Rachmilewitz D, Cohen P, Fich A Effect of chronic nicotine administration on trinitrobenzene sulphonic acid-induced colitis. Eur J Gastroenterol Hepatol 1998; 10: 1013-9.

11. Lin-Hua W, Zeng-Lai X, Di D, Shan-An H, Hong Y. Protective effect of anthocyanins extract from blueberry on TNBS-induced IBD model of mice. Evid Based Complement Alternat Med 2011; 2011: 5254-62.

12. Gonçalves H, Henriques-Coelho T, Rocha AP, Lourenço AP, Leite-Moreira A, Bernardes J. Comparison of different methods of heart rate entropy analysis during acute anoxia superimposed on a chronic rat model of pulmo nary hypertension. Med Eng Phys 2013; 35: 559-68.

13. Henze M, Hart D, Samarel A, Barakat J, Eckert L, Scrogin $\mathrm{K}$. Persistent alterations in heart rate variability, baroreflex sensitivity, and anxiety-like behaviors during development of heart failure in the rat. Am J Physiol Heart Circ Physiol 2008; 295: 29-38.

14. Zhou Q, Price DD, Caudle RM, Verne GN. Visceral and somatic hypersensitivity in TNBS induced colitis in rats. Dig Dis Sci 2008; 53; 429-35.

15. Holzer P, Michl T, Danzer M, Jocic M, Schicho R, Lippe IT. Surveillance of the gastrointestinal mucosa by sensory neurons. J Physiol Pharmacol 2001; 52: 505-21.

16. Geboes K, Collins S. Structural abnormalities of the nervous system in Crohn's disease and ulcerative colitis. Neurogastroenterol Motil 1998; 10: 189-202.

17. Schemann M. Control of gastrointestinal motility by the "gut brain" - the enteric nervous system. J Pediatr Gastroenterol Nutr 2005; 41 Suppl 1: 4-6.

18. Gershon MD. Review article: serotonin receptors and transporters: roles in normal and abnormal gastrointestinal motility. Aliment Pharmacol Ther 2004; 20 Suppl 7: 3-14

19. Woolf CJ, Salter MW. Neuronal plasticity: increasing the gain in pain. Science 2000; 288: 1765-9.

20. Neumann S, Doubell TP, Leslie T, Woolf CJ. Inflammatory pain hypersensitivity mediated by phenotypic switch in myelinated primary sensory neurons. Nature 1996; 384 360-4.

21. Lee JH, Kwon OD, Ahn SH, et al. Reduction of gastro intestinal motility by unilateral thyroparathyroidectomy plus subdiaphragmatic vagotomy in rats. World J Gastroenterol 2012; 18: 4570-7.

22. Chen S, Li J, Zhang L, et al. 5-HT 3 receptors mediate the time-dependent vagal afferent modulation of noci ception during chronic food allergen-sensitized visceral hyperalgesia in rats. Neurogastroenterol Motil 2009; 21 : 1222-e113.

23. Van Der Zanden EP, Boeckxstaens GE, De Jonge WJ. The vagus nerve as a modulator of intestinal inflammation. Neurogastroenterol Motil 2009; 21: 6-17.

24. Mazelin L, Theodorou V, More J, Fioramonti J, Bueno L. Protective role of vagal afferents in experimentally-induced colitis in rats. J Auton Nerv Syst 1998; 73: 38-45.

25. Wang $H$, Liao $H$, Ochani $M$, et al. Cholinergic agonists inhibit HMGB1 release and improve survival in experimental sepsis. Nat Med 2004; 10: 1216-21.

26. Ciesielczyk K, Zurowski D, Nowak $九$, Thor PJ. Vagal modulation of nociception in experimental model of viscreal pain. Folia Med Cracov 2008; 49: 79-89.

27. Pavithran P, Nandeesha H, Sathiyapriya V, Bobby Z, Madanmohan T. Short term heart variability and oxidative stress in newly diagnosed essential hypertension. Clin Exp Hyperten 2008; 30: 486-96.

28. Yarushkina NI, Bagaeva TR, Filaretova LP. Central corticotropin-releasing factor (CRF) may attenuate somatic pain sensitivity through involvement of glucocorticoids. J Physiol Pharmacol 2011; 62: 541-8.

29. Heart rate variability. Standards of measurement, physiological interpretation, and clinical use. Task Force of the European Society of Cardiology and the North American Society of Pacing and Electrophysiology. Eur Heart J 1996; 17: 354-81.

30. Yoon YZ, Kim J, Lee BC, Kim YU, Lee SK, Soh KS. Changes in ultraweak photon emission and heart rate variability of epinephrine-injected rats. Gen Physiol Biophys 2005; 24: 147-59.

31. Malliani A. Heart rate variability: from bench to bedside. Eur J Intern Med 2005; 16: 12-20.

32. McCafferty DM, Wallace JL, Sharkey KA. Effects of chemical sympathectomy and sensory nerve ablation on experimental colitis in the rat. Am J Physiol 1997; 272: 272-80.

33. Furlan R, Ardizzone S, Palazzolo L, et al. Sympathetic overactivity in active ulcerative colitis: effects of clonidine. Am J Physiol Regul Integr Comp Physiol 2006; 290: 224-32.

34. Straub RH, Herfarth H, Falk W, Andus T, Scholmerich J. Uncoupling of the sympathetic nervous system and the hypothalamic-pituitary-adrenal axis in inflammatory bowel disease? J Neuroimmunol 2002; 126: 116-25.

35. Sharma P, Makharia GK, Ahuja V, Dwivedi SN, Deepak KK. Autonomic dysfunctions in patients with inflammatory bowel disease in clinical remission. Dig Dis Sci 2009; 54 : 853-61.

36. Grover M, Drossman DA. Functional abdominal pain. Curr Gastroenterol Rep 2010; 12: 391-8.

37. Greenwood B, Mantle M. Mucin and protein release in the rabbit jejunum: effects of bethanechol and vagal nerve stimulation. Gastroenterology 1992; 103: 496-505. 